\title{
A New Modified Efficient Levenberg-Marquardt Method for Solving Systems of Nonlinear Equations
}

\author{
Zhenxiang Wu, ${ }^{1}$ Tong Zhou, ${ }^{1}$ Lei $\mathrm{Li}^{1}{ }^{1}$ Liang Chen $\mathbb{D}^{1,2}$ and Yanfang $\mathrm{Ma}^{3,4}$ \\ ${ }^{1}$ School of Mathematical Sciences, Huaibei Normal University, Huaibei 235000, China \\ ${ }^{2}$ School of Sciences, Changzhou Institute of Technology, Changzhou, Jiangsu 213032, China \\ ${ }^{3}$ School of Computer Science and Technology, Huaibei Normal University, Huaibei 235000, China \\ ${ }^{4}$ School of Computer Science and Information Engineering, Changzhou Institute of Technology, Changzhou, \\ Jiangsu 213032, China \\ Correspondence should be addressed to Liang Chen; clmyf2@163.com
}

Received 7 June 2021; Accepted 14 August 2021; Published 31 August 2021

Academic Editor: Lei Liu

Copyright (c) 2021 Zhenxiang Wu et al. This is an open access article distributed under the Creative Commons Attribution License, which permits unrestricted use, distribution, and reproduction in any medium, provided the original work is properly cited.

For systems of nonlinear equations, a modified efficient Levenberg-Marquardt method with new LM parameters was developed by Amini et al. (2018). The convergence of the method was proved under the local error bound condition. In order to enhance this method, using nonmonotone technique, we propose a new Levenberg-Marquardt parameter in this paper. The convergence of the new Levenberg-Marquardt method is shown to be at least superlinear, and numerical experiments show that the new Levenberg-Marquardt algorithm can solve systems of nonlinear equations effectively.

\section{Introduction}

Consider the system of nonlinear equations

$$
F(x)=0
$$

where the function $F(x): \mathbf{R}^{n} \longrightarrow \mathbf{R}^{m}$ is continuously differentiable. In this paper, we assume that the solution set of (1) (denoted by $X^{*}$ ) is nonempty, with $\|\cdot\|$ referring to the 2norm.

Newton method is an important method to solve system (1) in [1]. At each iteration, it uses the trial step

$$
d_{k}^{N}=-J_{k}^{-1} F_{k}
$$

where $F\left(x_{k}\right)=F_{k}$ and $J_{k}=F^{\prime}\left(x_{k}\right)$ is Jacobian matrix. When $J(x)$ is Lipschitz continuous and nonsingular, then the convergence of this method is quadratic at the solution. However, trial step $d_{k}^{N}$ may not exist and $J_{k}$ is singular or near singular. Newton method may not be well defined. To overcome this difficulty, Levenberg-Marquardt (LM) method was created by Levenberg [2] and Marquardt [3] which uses the trial step $d_{k}^{\mathrm{LM}}$ at each iteration, where

$$
d_{k}^{\mathrm{LM}}=-\left(J_{k}^{T} J_{k}+\lambda_{k} I\right)^{-1} J_{k}^{T} F_{k}
$$

and $\lambda_{k}$ is a nonnegative constant. By introducing a nonnegative parameter $\lambda_{k}$, LM method overcomes the problem that $J_{k}$ is singular or near singular; furthermore, excessive step size $\left\|d_{k}\right\|$ is avoided. In this case, where $\lambda_{k}=0$ and Jacobian matrix $J_{k}$ is nonsingular, the LM method is reduced to Newton method.

The efficiency of the LM method is affected by the parameter $\lambda_{k}$. For example, let $\lambda_{k}=\left\|F\left(x_{k}\right)\right\|^{2}$, under the local error bound condition, the LM method is shown to have quadratic convergence by Yamashita and Fukushima in [4]. However, when the sequence $\left\{x_{k}\right\}$ is far away from the set $X^{*},\left\|F_{k}\right\|$ may be very big which may lead to large $\lambda_{k}$. It will result in a smaller LM step size, further reducing the efficiency of the algorithm. In [5], Fan used $\lambda_{k}=\mu_{k}\left\|F\left(x_{k}\right)\right\|^{\delta}, \delta \in(1,2]$, where $\mu_{k}$ is updated with trust region technology in each iteration, the LM method also has quadratic convergence under some suitable conditions, and $\lambda_{k}=\mu_{k}\left\|F\left(x_{k}\right)\right\|^{\delta}$ can alleviate the effect of the initial point being far away from the set $X^{*}$. 
To avoid this trouble, Amini used $\lambda_{k}=\mu_{k}\left\|F_{k}\right\| /\left(1+\left\|F_{k}\right\|\right)$ in [6]; when the sequence $\left\{x_{k}\right\}$ is far from the solution set and $\left\|F_{k}\right\|$ is very large, $\lambda_{k}$ is close to $\mu_{k}$, which effectively controls the range of $\lambda_{k}$. Umar proposed some new LM parameters $\lambda_{k}=\left(\left\|J_{k}\right\| /\left\|J_{k}\right\|^{2}\right), \lambda_{k}=\left(\left\|J_{k}^{T} J_{k}\right\| /\right.$ $\left.\left\|J_{k}^{T} J_{k}\right\|^{2}\right)$ in [7]. Wang used $\lambda_{k}=\eta_{k}\left\|J_{k}^{T} F_{k}\right\|^{\alpha}$ with $\eta_{k}$ updated by trust region techniques from iteration to iteration in [8].

Ma introduced $\left\|J_{k}^{T} F_{k}\right\|$ into LM method and used a new LM parameter $\lambda_{k}=\theta\left\|F_{k}\right\|+(1-\theta)\left\|J_{k}^{T} F_{k}\right\|$ in [9], where $0 \leq \theta \leq 1$. It is noticeable that $\lambda_{k}$ is a convex combination of $\left\|F_{k}\right\|$ and $\left\|J_{k}^{T} F_{k}\right\|$, and the quadratic convergence of this method is proved. There are numerous other various LM methods to solve (1); interested readers are referred to [10-12] for related work. In order to discuss the range of parameter $\lambda_{k}$, inspired by $[6,8,9]$, in this paper, we choose a new LM parameter as follows:

$$
\lambda_{k}=\mu_{k}\left(\theta \frac{\left\|F_{k}\right\|^{\delta}}{1+\left\|F_{k}\right\|^{\delta}}+(1-\theta) \frac{\left\|J_{k}^{T} F_{k}\right\|^{\delta}}{1+\left\|J_{k}^{T} F_{k}\right\|^{\delta}}\right), \quad \delta \in(0,2], \theta \in[0,1],
$$

where $\lambda_{k}$ is a convex combination of $\left\|F_{k}\right\|^{\delta} /\left(1+\left\|F_{k}\right\|^{\delta}\right)$ and $\left\|J_{k}^{T} F_{k}\right\|^{\delta} /\left(1+\left\|J_{k}^{T} F_{k}\right\|^{\delta}\right)$ and $\mu_{k}$ is updated with trust region technology in each iteration.

Now, we set

$$
\phi(x)=\|F(x)\|^{2}
$$

as the merit function for (1). We define the actual reduction and the predicted reduction of $\phi(x)$ at the $k$ th iteration as follows:

$$
\begin{aligned}
& \operatorname{Ared}_{k}=\left\|F_{k}\right\|^{2}-\left\|F\left(x_{k}+d_{k}\right)\right\|^{2}, \\
& \operatorname{Pred}_{k}=\left\|F_{k}\right\|^{2}-\left\|F_{k}+J_{k} d_{k}\right\|^{2},
\end{aligned}
$$

where $d_{k}$ is computed by (3). The following ratio is

$$
r_{k}=\frac{\operatorname{Ared}_{k}}{\operatorname{Pred}_{k}}
$$

Grippo applied the nonmonotone line search technique to Newton's method in [13]; some authors have extended the nonmonotone techniques to trust region algorithm and proposed a lot of effective nonmonotone trust region methods in $[14,15]$. And Amini proposed nonmonotone line search technique for the LM method in [6]. Numerical experiments show that the algorithm with the nonmonotone technique is more efficient than the algorithm without the nonmonotone technique. Inspired by these theories, we apply a nonmonotone strategy to LM method in this paper. Let us replace actual reduction (6) with the following actual reduction:

$$
\overline{A r e d}_{k}=F_{l(k)}^{2}-\left\|F\left(x_{k}+d_{k}\right)\right\|^{2},
$$

where

$$
F_{l(k)}=\max _{0 \leq j \leq n(k)}\left\{\left\|F_{k-j}\right\|\right\}, \quad k=0,1, \ldots,
$$

$n(k)=\min \left\{N_{0}, k\right\}$, and $N_{0}$ is a positive integer constant. Obviously, by this change, $\left\|F_{k+1}\right\|$ will be compared with the $\max _{0 \leq j \leq n(k)}\left\{\left\|F_{k-j}\right\|\right\}$ in each iteration, further leading to affect the ratio. The ratio after the change is

$$
\bar{r}_{k}=\frac{\overline{\text { Ared }}_{k}}{\operatorname{Pred}_{k}} \text {. }
$$

It can be used to decide whether the trial step is accepted and update the trust region parameter $\mu_{k}$.

The paper is organized as follows. In Section 2, we present a new algorithm and then prove the global convergence of the new algorithm under some conditions. In Section 3, under the local error bound condition, the convergence of the new Levenberg-Marquardt method is shown to be at least superlinear. In Section 4, the new algorithm is an effective algorithm, which is demonstrated by numerical results. At last, we give some conclusions in Section 5 .

\section{The Efficient Algorithm and Global Convergence}

In this section, firstly, we present the new efficient LM algorithm and then prove the global convergence of the new algorithm.

When sequence $\left\{x_{k}\right\}$ is close to the solution, the steps may be too large, so we require

$$
\mu_{k} \geq m,
$$

in the new algorithm, where $m$ is a positive constant, and this is implemented by Step 5 .

Lemma 1. For all $k \in N$, we have

$$
\operatorname{Pred}_{k} \geq\left\|J_{k}^{T} F_{k}\right\| \min \left\{\left\|d_{k}\right\|, \frac{\left\|J_{k}^{T} F_{k}\right\|}{\left\|J_{k}^{T} J_{k}\right\|}\right\} .
$$

Proof. This proof is directly derived from the important theory given by Powell in [16].

From literature [6], the following lemma can be obtained.

Lemma 2. Suppose the sequence $\left\{x_{k}\right\}$ be generated by Algorithm 1, then the sequence $\left\{F_{l(k)}\right\}$ converges.

Next we present some of the assumptions needed in the following content.

\section{Assumption 1}

(a) $F(x)$ is continuously differentiable and Lipschitz continuous; i.e., there exists a positive constant $L_{2}$ that makes

$$
\|F(y)-F(x)\| \leq L_{2}\|y-x\| .
$$


(b) $J(x)$ is Lipschitz continuous; i.e., there exists a positive constant $L_{1}$ such that

$$
\|J(y)-J(x)\| \leq L_{1}\|y-x\| .
$$

Lemma 3. If Assumption 1 holds, then we have

$$
\begin{gathered}
\|F(y)-F(x)-J(x)(y-x)\| \leq L_{1}\|y-x\|^{2}, \\
J(x) \leq L_{2} .
\end{gathered}
$$

Proof. The proof of (17) can be found in [17]. So, we only prove (16). Using mean value theorem, there exists $z \in[x, y]$ that makes

$$
F(y)=F(x)+J(x)(y-x),
$$

and hence,

$$
F(y)-F(x)-J(x)(y-x)=(J(z)-J(x))(y-x) .
$$

According to the last equation, we can obtain

$$
\begin{aligned}
\|F(y)-F(x)-J(x)(y-x)\| & \leq\|(J(z)-J(x))\|\|(y-x)\| \\
& \leq L_{1}\|(z-x)\|\|(y-x)\| \\
& \leq L_{1}\|(y-x)\|^{2} .
\end{aligned}
$$

So (16) is true.

Theorem 1. Suppose that Assumption 1 is true. Then, Algorithm 1 terminates in finite iterations or satisfies

$$
\liminf _{k \longrightarrow \infty}\left\|J_{k}^{T} F_{k}\right\|=0 .
$$

Proof. Assume that the theorem is incorrect; then, there exist a positive constant $\varepsilon_{0}$ and a constant $k_{0} \in N$ that makes

$$
\left\|J_{k}^{T} F_{k}\right\| \geq \varepsilon_{0}, \quad \forall k \geq k_{0} .
$$

Firstly, we prove that

$$
\lim _{k \rightarrow \infty}\left\|F\left(x_{l(k)}\right)\right\|=\lim _{k \longrightarrow \infty}\left\|F\left(x_{k}\right)\right\| .
$$

Since $d_{k}$ is accepted by the algorithm, we have

$$
F_{l(k)}^{2}-\left\|F\left(x_{k}+d_{k}\right)\right\|^{2} \geq p_{0} \operatorname{Pred}_{k} .
$$

This, along with (17), (22), and Lemma 1 , for all $k \geq k_{0}$, that means

$$
\begin{aligned}
F_{l(k)}^{2}-\left\|F_{k+1}\right\|^{2} & \geq p_{0}\left\|J_{k}^{T} F_{k}\right\| \min \left\{\left\|d_{k}\right\|, \frac{\left\|J_{k}^{T} F_{k}\right\|}{\left\|J_{k}^{T} J_{k}\right\|}\right\} \\
& \geq p_{0} \varepsilon_{0} \min \left\{\left\|d_{k}\right\|, \frac{\varepsilon_{0}}{L_{2}^{2}}\right\} .
\end{aligned}
$$

Replacing $k$ with $l(k)-1$, for all sufficiently large $k$, there is

$$
F_{l(l(k)-1)}^{2}-\left\|F_{l(k)}\right\|^{2} \geq p_{0} \min \left\{\left\|d_{l(k)-1}\right\|, \frac{\varepsilon_{0}}{L_{2}^{2}}\right\} .
$$

From Lemma 2

$$
\lim _{k \longrightarrow \infty}\left(\left\|F_{l(l(k)-1)}^{2}\right\|-\left\|F_{l(k)}\right\|^{2}\right)=0,
$$

which together with the last inequality yields

$$
\lim _{k \longrightarrow \infty} \min \left\{\left\|d_{l(k)-1}\right\|, \frac{\varepsilon_{0}}{L_{2}^{2}}\right\} .
$$

$\varepsilon_{0} / L_{1}^{2}$ is a positive constant, so

$$
\lim _{k \longrightarrow \infty}\left\|d_{l(k)-1}\right\|=0 .
$$

Using Assumption 1, the last equality implies that

$$
\lim _{k \longrightarrow \infty}\left\|F\left(x_{l(k)}\right)\right\|=\lim _{k \longrightarrow \infty}\left\|F\left(x_{l(k)-1}\right)\right\| .
$$

Let $\widehat{l}(k)=l\left(k+N_{0}+2\right)$. Using induction, for all $j \geq 1$, we can show that

$$
\lim _{k \longrightarrow \infty}\left\|d_{\hat{l}(k)-j}\right\|=0 .
$$

For $j=1$, we can have from (31) that (29) is true. Assuming that (29) is true for given $j$, we show that (29) holds for given $j+1$. Let $k$ be large enough such that $\widehat{l}(k)-(j+1)>0$. Substituting $k$ with $\widehat{l}(k)-j-1$ and using (24), we obtain

$$
F_{l(\widehat{l}(k)-j-1)}^{2}-\| F\left(x_{\hat{l}(k)-j} \|^{2} \geq p_{0} \operatorname{Pred}_{l(k)-j-1} .\right.
$$

Similarly, we can deduce that

$$
\lim _{k \longrightarrow \infty}\left\|d_{l(k)-j-1}\right\|=0 .
$$

Therefore (31) holds. Along with Assumption 1 we imply that

$$
\lim _{k \longrightarrow \infty}\left\|F\left(x_{\hat{l}(k)-j-1}\right)\right\|=\lim _{k \longrightarrow \infty}\left\|F\left(x_{\hat{l}(k)-j}\right)\right\| .
$$

Similarly, for any given $j \geq 1$, we have $\lim _{k \rightarrow \infty}$ $\left\|F\left(x_{l(k)-j}\right)\right\|=\lim _{k \rightarrow \infty}\left\|F\left(x_{l(k)}\right)\right\|$. On the one hand, for any $k$, we have

$$
x_{k+1}=x_{\hat{l}(k)}-\sum_{j=1}^{\hat{l}(k)-k-1} d_{\widehat{l}(k)-j^{\prime}}
$$

Using (31) and the fact that $\widehat{l}(k)-j-1 \leq N_{0}+1$, we have

$$
\lim _{k \longrightarrow \infty}\left\|x_{k+1}-x_{\hat{l}(k)}\right\|=0,
$$

With Assumption 1, we conclude

$$
\lim _{k \longrightarrow \infty}\left\|F\left(x_{l(k)}\right)\right\|=\lim _{k \longrightarrow \infty}\left\|F\left(x_{\hat{l}(k)}\right)\right\|=\lim _{k \longrightarrow \infty}\left\|F\left(x_{k}\right)\right\| .
$$


And then (22) is proved. By using (22) and (25), we can deduce that

$$
\lim _{k \rightarrow \infty}\left\|d_{k}\right\|=0 .
$$
that

Then, it follows from (3) in Algorithm 1, (17), and (22)

$$
\mu_{k} \longrightarrow \infty \text {, as } k \longrightarrow \infty \text {. }
$$

On the other hand, by (6), (8), (17), (22), (38), and Lemma 1, we can deduce that

$$
\begin{aligned}
r_{k} & =\frac{\operatorname{Ared}_{k}}{\operatorname{Pred}_{k}} \\
& =\frac{\left\|F_{k}\right\|^{2}-\left\|F_{k+1}\right\|^{2}}{\operatorname{Pred}_{k}} \\
& =1+\frac{\left\|F_{k}+J_{k} d_{k}\right\| O\left(\left\|d_{k}\right\|^{2}\right)+O\left(\left\|d_{k}\right\|^{4}\right)}{\operatorname{Pred}_{k}} \\
& \leq 1+\frac{\left\|F_{k}+J_{k} d_{k}\right\| O\left(\left\|d_{k}\right\|^{2}\right)+O\left(\left\|d_{k}\right\|^{4}\right)}{\left\|J_{k}^{T} F_{k}\right\| \min ^{4}\left(\left\|d_{k}\right\|,\left(\left\|J_{k}^{T} F_{k}\right\| /\left\|J_{k}^{T} J_{k}\right\|\right)\right)} \\
& \leq 1+\frac{O\left(\left\|d_{k}\right\|^{2}\right)}{\left\|d_{k}\right\|} \longrightarrow 1 .
\end{aligned}
$$

And then combined with (8) and (10), we can see that

$$
\begin{aligned}
\bar{r}_{k} & =\frac{\overline{A r e d}_{k}}{\operatorname{Pred}_{k}}=\frac{F_{l(k)}^{2}-\left\|F\left(x_{k}+d_{k}\right)\right\|^{2}}{\operatorname{Pred}_{k}} \geq \frac{F_{k}^{2}-\left\|F_{k+1}\right\|^{2}}{\operatorname{Pred}_{k}} \\
& =r_{k} \longrightarrow 1 .
\end{aligned}
$$

Considering Algorithm 1, obviously, for all large $k$, there exists a positive constant $\bar{\mu}>m$ such that $\mu_{k}<\bar{\mu}$, which conflicts with (22) and so Theorem 1 is true.

\section{Local Convergence}

Definition 1. Let $N$ be a subset of $\mathbf{R}^{n}$ that makes $N \cap X^{*} \neq \varnothing$. We say $\|F(x)\|$ provides a local error bounded on $N$ for (1), where $\left\|x_{k}-\bar{x}_{k}\right\|=\operatorname{dist}\left(x_{k}, X^{*}\right)$, if there exists a positive constant $C_{1}$ such that

$$
\|F(x)\| \geq C_{1} \operatorname{dist}\left(x, X^{*}\right), \quad \forall x \in N .
$$

\section{Assumption 2}

(a) $F(x)$ is continuously differentiable, and $\|F(x)\|$ provides a local error bound on subset $N\left(x^{*}, b\right)$ for problem (1), where

$$
N\left(x^{*}, b\right)=\left\{x \in \mathbf{R}^{n} \mid\left\|x-x^{*}\right\| \leq b\right\}, \quad 0<b<1 .
$$

(b) $F(x)$ and $J(x)$ are both Lipschitz continuous on $N\left(x^{*}, b\right)$; that is, there exist two positive constants $L_{1}$, $L_{2}$ that make

$$
\begin{gathered}
\|J(y)-J(x)\| \leq L_{1}\|y-x\|, \quad \forall x, y \in N\left(x^{*}, b\right), \\
\|F(y)-F(x)\| \leq L_{2}\|y-x\|, \quad \forall x, y \in N\left(x^{*}, b\right),
\end{gathered}
$$

which implies

$$
\begin{aligned}
& \|F(y)-F(x)-J(x)(y-x)\| \leq L_{1}\|y-x\|^{2}, \\
& \forall x, y \in N\left(x^{*}, b\right) .
\end{aligned}
$$

Lemma 4. Suppose that Assumption 2 is true. Then, for all sufficiently large $k$, we have the following.

(1) There exists a positive constant $M>m$ that makes

$$
\mu_{k} \leq M
$$

(2) $\lambda_{k} \geq C_{3}\left\|x_{k}-\bar{x}_{k}\right\|^{\delta}$, where $C_{3}=\left(C_{1}^{\delta} / 2\right) m \theta+\left(C_{2}^{\delta} / 2\right)$ $m(1-\theta)$.

Proof. The proof process of (1) is the same as that of Lemma 3.2 in [6], so we are not going to prove it here and only give the proof of (2).

$$
\left\|F_{k}\right\|^{2}=F_{k}^{T} F_{k}=F_{k}^{T}\left(F\left(\bar{x}_{k}\right)+J_{k}\left(x_{k}-\bar{x}_{k}\right)\right)+F_{k}^{T} V_{k},
$$

where $V_{k}=F_{k}-\bar{F}_{k}-J_{k}\left(x_{k}-\bar{x}_{k}\right)$, obviously,

$$
F_{k}^{T} J_{k}\left(x_{k}-\bar{x}_{k}\right)=\left\|F_{k}\right\|^{2}-F_{k}^{T} V_{k} \text {. }
$$

We can obtain from Definition 1 and (45) and (46) that

$$
\begin{aligned}
\left\|J_{k}^{T} F_{k}\right\|\left\|x_{k}-\bar{x}_{k}\right\| & \geq C_{1}^{2}\left\|x_{k}-\bar{x}_{k}\right\|^{2}-L_{1} L_{2}\left\|x_{k}-\bar{x}_{k}\right\|^{3}, \\
\left\|J_{k}^{T} F_{k}\right\| & \geq C_{1}^{2}\left\|x_{k}-\bar{x}_{k}\right\|-L_{1} L_{2}\left\|x_{k}-\bar{x}_{k}\right\|^{2} \\
& \geq C_{2}\left\|x_{k}-\bar{x}_{k}\right\| .
\end{aligned}
$$

Thus we obtain

$$
\left\|J_{k}^{T} F_{k}\right\|^{\delta} \geq C_{2}^{\delta}\left\|x_{k}-\bar{x}_{k}\right\|^{\delta}
$$

Then we show the following inequalities:

$$
\begin{gathered}
\frac{\left\|F_{k}\right\|^{\delta}}{1+\left\|F_{k}\right\|^{\delta}} \geq \frac{C_{1}^{\delta}}{2}\left\|x_{k}-\bar{x}_{k}\right\|^{\delta}, \\
\frac{\left\|J_{k}^{T} F_{k}\right\|^{\delta}}{1+\left\|J_{k}^{T} F_{k}\right\|^{\delta}} \geq \frac{C_{2}^{\delta}}{2}\left\|x_{k}-\bar{x}_{k}\right\|^{\delta} .
\end{gathered}
$$

If $\left\|F_{k}\right\| \leq 1$, then the following holds:

$$
\frac{\left\|F_{k}\right\|^{\delta}}{1+\left\|F_{k}\right\|^{\delta}} \geq \frac{C_{1}^{\delta}}{2}\left\|x_{k}-\bar{x}_{k}\right\|^{\delta},
$$

and if $\left\|F_{k}\right\|>1$, then 


$$
\frac{\left\|F_{k}\right\|^{\delta}}{1+\left\|F_{k}\right\|^{\delta}}>\frac{1}{2} .
$$

Through the above two inequalities, we have

$$
\frac{\left\|F_{k}\right\|^{\delta}}{1+\left\|F_{k}\right\|^{\delta}} \geq \max \left\{\frac{1}{2}, \frac{C_{1}^{\delta}}{2}\left\|x_{k}-\bar{x}_{k}\right\|^{\delta}\right\} .
$$

Similarly, if $\left\|J_{k}^{T} F_{k}\right\| \leq 1$, then the following holds

$$
\frac{\left\|J_{k}^{T} F_{k}\right\|^{\delta}}{1+\left\|J_{k}^{T} F_{k}\right\|^{\delta}} \geq \frac{\left\|J_{k}^{T} F_{k}\right\|^{\delta}}{2} \geq \frac{C_{2}^{\delta}}{2}\left\|x_{k}-\bar{x}_{k}\right\|^{\delta},
$$

and if $\left\|J_{k}^{T} F_{k}\right\|>1$, we have

$$
\frac{\left\|J_{k}^{T} F_{k}\right\|^{\delta}}{1+\left\|J_{k}^{T} F_{k}\right\|^{\delta}}>\frac{1}{2} .
$$

Hence, we obtain

$$
\frac{\left\|J_{k}^{T} F_{k}\right\|^{\delta}}{1+\left\|J_{k}^{T} F_{k}\right\|^{\delta}} \geq \max \left\{\frac{1}{2}, \frac{C_{2}^{\delta}}{2}\left\|x_{k}-\bar{x}_{k}\right\|^{\delta}\right\} .
$$

From Algorithm 1, (52), and (58), we have

$$
\begin{aligned}
\lambda_{k} & =\mu_{k}\left(\theta \frac{\left\|F_{k}\right\|^{\delta}}{1+\left\|F_{k}\right\|^{\delta}}+(1-\theta) \frac{\left\|J_{k}^{T} F_{k}\right\|^{\delta}}{1+\left\|J_{k}^{T} F_{k}\right\|^{\delta}}\right) \\
& \geq \frac{C_{1}^{\delta}}{2} m \theta\left\|x_{k}-\bar{x}_{k}\right\|^{\delta}+\frac{C_{2}^{\delta}}{2} m(1-\theta)\left\|x_{k}-\bar{x}_{k}\right\|^{\delta} \geq C_{3}\left\|x_{k}-\bar{x}_{k}\right\|^{\delta},
\end{aligned}
$$

where $C_{3}=\left(C_{1}^{\delta} / 2\right) m \theta+\left(C_{2}^{\delta} / 2\right) m(1-\theta)$. So when $\left\|x_{k}-\bar{x}_{k}\right\|$ is sufficiently small,

$$
\lambda_{k} \geq C_{3}\left\|x_{k}-\bar{x}_{k}\right\|^{\delta} .
$$

Lemma 5. Suppose that Assumption 2 is true, for all sufficiently large $k$. $\left\|d_{k}\right\|$ computed by (3) satisfies

$$
\left\|d_{k}\right\| \leq \mathrm{O}\left(\left\|x_{k}-\bar{x}_{k}\right\|\right) \text {. }
$$

Proof. If we set $\varphi_{k}(d)=\left\|F_{k}+J_{k} d\right\|^{2}+\lambda_{k}\|d\|^{2}$, then we have from (3) that $d_{k}$ is a minimizer of $\varphi_{k}(d)$, so it follows from Algorithm 1, (12), (45), (46), and $\left\|F\left(\bar{x}_{k}\right)\right\|=0$ that

$$
\begin{aligned}
\left\|d_{k}\right\|^{2} & \leq \frac{1}{\lambda_{k}} \varphi_{k}\left(x_{k}-\bar{x}_{k}\right) \\
& =\frac{1}{\lambda_{k}}\left(\left\|F_{k}+J_{k}\left(x_{k}-\bar{x}_{k}\right)\right\|^{2}+\lambda_{k}\left\|x_{k}-\bar{x}_{k}\right\|^{2}\right) \\
& \leq \frac{1}{C_{3}\left\|x_{k}-\bar{x}_{k}\right\|^{\delta}}\left(\left\|F_{k}+J_{k}\left(x_{k}-\bar{x}_{k}\right)\right\|^{2}+\lambda_{k}\left\|x_{k}-\bar{x}_{k}\right\|^{2}\right) \\
& \leq \frac{L_{1}^{2}\left\|x_{k}-\bar{x}_{k}\right\|^{4}}{C_{3}\left\|x_{k}-\bar{x}_{k}\right\|^{\delta}}+\left\|x_{k}-\bar{x}_{k}\right\|^{2} \\
& =O\left(\left\|x_{k}-\bar{x}_{k}\right\|^{2}\right) .
\end{aligned}
$$

So there is

$$
\left\|d_{k}\right\| \leq O\left(\left\|x_{k}-\bar{x}_{k}\right\|\right)
$$

Lemma 6. Suppose that Assumption 2 is true, for all sufficiently large $k$. So, we have

$$
\lambda_{k} \leq C_{4}\left\|x_{k}-\bar{x}_{k}\right\|^{\delta}
$$

Proof. Firstly, we deal with these two equations:

$$
\begin{aligned}
& \frac{\left\|F_{k}\right\|^{\delta}}{1+\left\|F_{k}\right\|^{\delta}} \leq\left\|F_{k}\right\|^{\delta} \leq L_{2}^{\delta}\left\|x_{k}-\bar{x}_{k}\right\|^{\delta}, \\
& \left\|J_{k}^{T} F_{k}\right\| \leq\left\|J_{k}^{T}\right\|\left\|F_{k}\right\| \leq L_{2}\left\|F\left(x_{k}\right)-F\left(\bar{x}_{k}\right)\right\| \leq L_{2}^{2}\left\|x_{k}-\bar{x}_{k}\right\| .
\end{aligned}
$$

On the other hand,

$$
\frac{\left\|J_{k}^{T} F_{k}\right\|^{\delta}}{1+\left\|J_{k}^{T} F_{k}\right\|^{\delta}} \leq\left\|J_{k}^{T} F_{k}\right\|^{\delta} \leq L_{2}^{2 \delta}\left\|x_{k}-\bar{x}_{k}\right\|^{\delta} .
$$

We conclude

$$
\begin{aligned}
\lambda_{k} & =\mu_{k}\left(\theta \frac{\left\|F_{k}\right\|^{\delta}}{1+\left\|F_{k}\right\|^{\delta}}+(1-\theta) \frac{\left\|J_{k}^{T} F_{k}\right\|^{\delta}}{1+\left\|J_{k}^{T} F_{k}\right\|^{\delta}}\right) \\
& \leq M\left(\theta L_{2}^{\delta}\left\|x_{k}-\bar{x}_{k}\right\|^{\delta}+(1-\theta) L_{2}^{2 \delta}\left\|x_{k}-\bar{x}_{k}\right\|^{\delta}\right) \\
& \leq C_{4}\left\|x_{k}-\bar{x}_{k}\right\|^{\delta},
\end{aligned}
$$

where $C_{4}=M \theta L_{2}^{\delta}+M(1-\theta) L_{1}^{\delta} L_{2}^{\delta}$. 
Without loss of generality, for all $\bar{x} \in N\left(x^{*}, b\right) \cap X^{*}$, suppose that $\operatorname{rank}(J(\bar{x}))=r$, and we prove the local convergence of Algorithm 1 by singular value decomposition (SVD) of $J(\bar{x})$.

$$
J(\bar{x})=\left[\begin{array}{ll}
\bar{U}_{1} & \bar{U}_{2}
\end{array}\right]\left[\begin{array}{cc}
\bar{\Sigma}_{1} & \\
& 0
\end{array}\right]\left[\begin{array}{ll}
\bar{V}_{1}^{T}, & \bar{V}_{2}^{T}
\end{array}\right],
$$

where $\bar{\Sigma}_{1}=\operatorname{diag}\left(\bar{\sigma}_{1}, \ldots, \bar{\sigma}_{r}\right) \quad$ with $\quad \bar{\sigma}_{1} \geq \bar{\sigma}_{2} \geq \cdots \geq \bar{\sigma}_{r}>0$, $\operatorname{rank}\left(\bar{\Sigma}_{1}\right)=r, \bar{U}=\left[\bar{U}_{1}, \bar{U}_{2}\right]$, and $\bar{V}=\left[\bar{V}_{1}, \bar{V}_{2}\right]$. And assume the SVD of $J(x)$ are as follows:

$$
\begin{aligned}
J(x)=U \Sigma V^{T} & =\left[U_{1}, U_{2}, U_{3}\right]\left[\begin{array}{lll}
\Sigma_{1} & & \\
& \Sigma_{2} & \\
& & 0
\end{array}\right]\left[\begin{array}{c}
V_{1}^{T} \\
V_{2}^{T} \\
V_{3}^{T}
\end{array}\right] \\
& =U_{1} \Sigma_{1} V_{1}^{T}+U_{2} \Sigma_{2} V_{2}^{T},
\end{aligned}
$$

where $U=\left[U_{1}, U_{2}, U_{3}\right]$ and $V=\left[V_{1}, V_{2}, V_{3}\right]$ are two orthogonal matrixes. $\Sigma_{1}=\operatorname{diag}\left(\sigma_{1}, \sigma_{2}, \ldots, \sigma_{r}\right), \sigma_{1} \geq \sigma_{2} \geq \cdots \geq$ $\sigma_{r}>0, \quad$ and $\quad \Sigma_{2}=\operatorname{diag}\left(\sigma_{r+1}, \sigma_{r+2}, \ldots, \sigma_{r+q}\right), \quad \sigma_{r+1} \geq \sigma_{r+2}$ $\geq \cdots \geq \sigma_{r+q}>0$.

Since $J(x)$ is Lipschitz continuous, by the theory of matrix perturbation [18], we have

$$
\left\|\operatorname{diag}\left(\Sigma_{1}-\bar{\Sigma}_{1}, \Sigma_{2}, 0\right)\right\| \leq\left\|J_{k}-\bar{J}_{k}\right\| \leq L_{1}\left\|x_{k}-\bar{x}_{k}\right\| .
$$

So there is

$$
\begin{aligned}
\left\|\Sigma_{1}-\bar{\Sigma}_{1}\right\| & \leq L_{1}\left\|\left(x_{k}-\bar{x}_{k}\right)\right\|, \\
\left\|\Sigma_{2}\right\| & \leq\left\|J_{k}-\bar{J}_{k}\right\| \leq L_{1}\left\|\left(x_{k}-\bar{x}_{k}\right)\right\| .
\end{aligned}
$$

Since $\left\{x_{k}\right\}$ converges to the set $X^{*}$, then we have $L_{1} \|\left(x_{k}-\right.$ $\left.\bar{x}_{k}\right) \| \leq\left(\bar{\sigma}_{r} / 2\right)$ hold for all sufficiently large $k$. So combined with (71), there is

$$
\left\|\Sigma_{1}^{-1}\right\| \leq \frac{1}{\bar{\sigma}_{r}-L_{1}\left\|\left(x_{k}-\bar{x}_{k}\right)\right\|} \leq \frac{2}{\bar{\sigma}_{r}} .
$$

Lemma 7. Suppose Assumption 2 holds; for all sufficiently large $k$, we have

(1) $\left\|U_{1} U_{1}^{T} F_{k}\right\| \leq O\left(\left\|x_{k}-\bar{x}_{k}\right\|\right)$

(2) $\left\|U_{2} U_{2}^{T} F_{k}\right\| \leq O\left(\left\|x_{k}-\bar{x}_{k}\right\|^{2}\right)$

Proof. The proof process is similar to Lemma 7 in [9], so we omit it here.

Theorem 2. Under Assumption 2, let $\left\{x_{k}\right\}$ be a sequence generated by Algorithm 1 with trust region technique. If $\delta \in(0,1)$, then $\left\{x_{k}\right\}$ converges superlinearly to the solution. If $\delta \in[1,2]$, then sequence $\left\{x_{k}\right\}$ converges quadratically to the solution.

Proof. Using (3) and (69), we obtain

$$
\begin{aligned}
d_{k} & =-V_{1}\left(\Sigma_{1}^{2}+\lambda_{k} I\right)^{-1} \Sigma_{1} U_{1}^{T} F_{k}-V_{2}\left(\Sigma_{2}^{2}+\lambda_{k} I\right)^{-1} \Sigma_{2} U_{2}^{T} F_{k}, \\
F_{k}+J_{k} d_{k} & =F_{k}-U_{1} \Sigma_{1}\left(\Sigma_{1}^{2}+\lambda_{k} I\right)^{-1} \Sigma_{1} U_{1}^{T} F_{k}-U_{2} \Sigma_{2}\left(\Sigma_{2}^{2}+\lambda_{k} I\right)^{-1} \Sigma_{2} U_{2}^{T} F_{k} \\
& =\lambda_{k} U_{1}\left(\Sigma_{1}^{2}+\lambda_{k} I\right)^{-1} U_{1}^{T} F_{k}+\lambda_{k} U_{2}\left(\Sigma_{2}^{2}+\lambda_{k} I\right)^{-1} U_{2}^{T} F_{k} .
\end{aligned}
$$

From (47), (71), Definition 1, and Lemma 5, we have

$$
\begin{aligned}
\left\|F_{k}+J_{k} d_{k}\right\| & \leq \lambda_{k}\left\|U_{1} U_{1}^{T} F_{k}\right\|+\left\|U_{2} U_{2}^{T} F_{k}\right\| \\
& \leq C_{4}\left\|x_{k}-\bar{x}_{k}\right\|^{\delta} O\left(\left\|x_{k}-\bar{x}_{k}\right\|\right)+O\left(\left\|x_{k}-\bar{x}_{k}\right\|^{2}\right) \\
& \leq C_{4}\left\|x_{k}-\bar{x}_{k}\right\|^{1+\delta}+O\left(\left\|x_{k}-\bar{x}_{k}\right\|^{2}\right) \\
& \leq O\left(\left\|x_{k}-\bar{x}_{k}\right\|^{1+\delta}\right)+O\left(\left\|x_{k}-\bar{x}_{k}\right\|^{2}\right) .
\end{aligned}
$$

From (44), (46), (74), Definition 1, and Lemma 5, we conclude that

$$
\begin{aligned}
C_{1}\left\|\left(x_{k+1}-\bar{x}_{k+1}\right)\right\| & \leq\left\|F\left(x_{k+1}\right)\right\|=\left\|F\left(x_{k}+d_{k}\right)\right\| \\
& \leq O\left(\left\|\left(x_{k}-\bar{x}_{k}\right)\right\|^{1+\delta}\right)+O\left(\left\|\left(x_{k}-\bar{x}_{k}\right)\right\|^{2}\right) .
\end{aligned}
$$

On the other hand, it is obvious that

$$
\left\|x_{k}-\bar{x}_{k}\right\|=\operatorname{dist}\left(x_{k}, X^{*}\right) \leq\left\|x_{k+1}-\bar{x}_{k}\right\| \leq\left\|x_{k+1}-\bar{x}_{k+1}\right\|+d_{k} \text {. }
$$

It follows from (75) and Lemma 5 that $\left\|x_{k}-\bar{x}_{k}\right\| \leq 2\left\|d_{k}\right\| \leq O\left(\left\|x_{k}-\bar{x}_{k}\right\|\right)$ holds for all sufficiently large $k$. So, $\left\|d_{k}\right\|=O\left(\left\|x_{k}-\bar{x}_{k}\right\|\right)$, and this is related to (75). We deduce that if $\delta \in(0,1),\left\|d_{k+1}\right\|=O\left(\left\|d_{k}\right\|^{1+\delta}\right)$. If $\delta \in[1,2],\left\|d_{k+1}\right\|=O\left(\left\|d_{k}\right\|^{2}\right)$. So if $\delta \in(0,1),\left\{x_{k}\right\}$ converges superlinearly to the solution; otherwise, if $\delta \in[1,2],\left\{x_{k}\right\}$ converges quadratically to the solution.

\section{Numerical Experiments}

In Section 5, we compare the performance of Algorithm 1 with Algorithm 2.1 (writing Algorithm 2.1 as AELM) in [6] through some numerical experiments. The test function $F(\bar{x})$ is improved by the method in [19]. The form is as follows:

$$
F(\bar{x})=F(x)-J\left(x^{*}\right) A\left(A^{T} A\right)^{-1} A^{T}(x-x),
$$


Input: given $x_{0} \in \mathbf{R}^{n}, N_{0}>0, \mu_{0}>m>0, \varepsilon>0,0<p_{0}<p_{1}<p_{2}<1, k:=0$

\section{Output:}

Step 1. If $J_{k}^{T} F_{k} \leq \varepsilon$, stop. Otherwise, set

$\lambda_{k}=\mu_{k}\left(\theta\left\|F_{k}\right\|^{\delta} /\left(1+\left\|F_{k}\right\|^{\delta}\right)+(1-\theta)\left\|J_{k}^{T} F_{k}\right\|^{\delta} /\left(1+\left\|J_{k}^{T} F_{k}\right\|^{\delta}\right)\right)$,

where $\theta \in[0,1], \delta \in(0,2]$.

Step 2. Compute the search direction

$d_{k}=\left(J_{k}^{T} J_{k}+\lambda_{k} I\right)^{-1} J_{k}^{T} F_{k}$.

Step 3. By (7), (8), and (10), compute $\operatorname{Pred}_{k}, \overline{A r e d}_{k}, \bar{r}_{k}$.

Step 4. Set

$x_{k+1}= \begin{cases}x_{k}+d_{k}, & \bar{r}_{k} \geq p_{0} \\ x_{k}, & \bar{r}_{k}<p_{0}\end{cases}$

Step 5. Choose $\mu_{k+1}$ as

$$
\mu_{k+1}= \begin{cases}4 \mu_{k}, & \bar{r}_{k}<p_{1} \\ \mu_{k}, & \bar{r}_{k} \in\left[p_{1}, p_{2}\right] \\ \max \left\{\left(\mu_{k} / 4\right), m\right\}, & \bar{r}_{k}>p_{2}\end{cases}
$$

Step 6. Set $k:=k+1$, and go to Step 1 .

Algorithm 1: A modified efficient Levenberg-Marquardt algorithm.

TABLE 1: Results on singular nonlinear equations with $\operatorname{rank}\left(F^{\prime}\left(x^{*}\right)=n-1\right.$.

\begin{tabular}{|c|c|c|c|c|c|c|c|c|}
\hline \multirow{3}{*}{ Problem } & \multirow{3}{*}{$n$} & \multirow{3}{*}{$x_{0}$} & \multicolumn{6}{|c|}{$\mathrm{NF} / \mathrm{NJ}$} \\
\hline & & & \multirow{2}{*}{ AELM } & \multicolumn{2}{|c|}{$\begin{array}{l}\text { Algorithm with } \\
\qquad \delta=1\end{array}$} & \multicolumn{3}{|c|}{ Algorithm with $\delta=2$} \\
\hline & & & & $\theta=0$ & $\theta=0.5$ & $\theta=0$ & $\theta=0.5$ & $\theta=1$ \\
\hline \multirow{6}{*}{ Helical valley function } & \multirow{6}{*}{3} & -100 & $7 / 7$ & $7 / 7$ & $8 / 8$ & $9 / 9$ & $7 / 7$ & $7 / 7$ \\
\hline & & -10 & $6 / 6$ & $6 / 6$ & $7 / 7$ & $7 / 7$ & $6 / 6$ & $6 / 6$ \\
\hline & & -1 & $1 / 1$ & $1 / 1$ & $1 / 1$ & $1 / 1$ & $1 / 1$ & $1 / 1$ \\
\hline & & 1 & $9 / 9$ & $9 / 9$ & $9 / 9$ & $9 / 9$ & $9 / 9$ & $9 / 9$ \\
\hline & & 10 & $8 / 8$ & $8 / 8$ & $8 / 8$ & $9 / 9$ & $8 / 8$ & $8 / 8$ \\
\hline & & 100 & $8 / 8$ & $8 / 8$ & $8 / 8$ & $8 / 8$ & $8 / 8$ & $8 / 8$ \\
\hline \multirow{18}{*}{ Discrete boundary value } & \multirow{6}{*}{10} & -100 & $14 / 14$ & $14 / 14$ & $14 / 14$ & $14 / 14$ & $14 / 14$ & $14 / 14$ \\
\hline & & -10 & $11 / 11$ & $10 / 10$ & $11 / 11$ & $10 / 10$ & $10 / 10$ & $10 / 10$ \\
\hline & & -1 & $8 / 8$ & $8 / 8$ & $8 / 8$ & $7 / 7$ & $7 / 7$ & $7 / 7$ \\
\hline & & 1 & $3 / 3$ & $3 / 3$ & $3 / 3$ & $4 / 4$ & $4 / 4$ & $4 / 4$ \\
\hline & & 10 & $11 / 11$ & $10 / 10$ & $10 / 10$ & $9 / 9$ & $9 / 9$ & $9 / 9$ \\
\hline & & 100 & $9 / 9$ & $9 / 9$ & $9 / 9$ & $9 / 9$ & $9 / 9$ & $9 / 9$ \\
\hline & \multirow{6}{*}{100} & -100 & $14 / 14$ & $13 / 13$ & $14 / 14$ & $13 / 13$ & $14 / 14$ & $14 / 14$ \\
\hline & & -10 & $11 / 11$ & $9 / 9$ & $11 / 11$ & $8 / 8$ & $9 / 9$ & $9 / 9$ \\
\hline & & -1 & $12 / 12$ & $5 / 5$ & $5 / 5$ & $4 / 4$ & $4 / 4$ & $4 / 4$ \\
\hline & & 1 & $4 / 4$ & $4 / 4$ & $4 / 4$ & $3 / 3$ & $3 / 3$ & $3 / 3$ \\
\hline & & 10 & $7 / 7$ & $7 / 7$ & $7 / 7$ & $7 / 7$ & $5 / 5$ & $8 / 8$ \\
\hline & & 100 & $12 / 12$ & $11 / 11$ & $12 / 12$ & $11 / 11$ & $12 / 12$ & $12 / 12$ \\
\hline & \multirow{6}{*}{500} & -100 & $15 / 15$ & $13 / 13$ & $15 / 15$ & $12 / 12$ & $14 / 14$ & $15 / 15$ \\
\hline & & -10 & $8 / 8$ & $6 / 6$ & $7 / 7$ & $5 / 5$ & $6 / 6$ & $6 / 6$ \\
\hline & & -1 & $6 / 6$ & $5 / 5$ & $5 / 5$ & $4 / 4$ & $4 / 4$ & $4 / 4$ \\
\hline & & 1 & $1 / 1$ & $1 / 1$ & $1 / 1$ & $1 / 1$ & $1 / 1$ & $1 / 1$ \\
\hline & & 10 & $8 / 8$ & $6 / 6$ & $7 / 7$ & $5 / 5$ & $6 / 6$ & $6 / 6$ \\
\hline & & 100 & $15 / 15$ & $12 / 12$ & $14 / 14$ & $11 / 11$ & $13 / 13$ & $14 / 14$ \\
\hline
\end{tabular}


TABLE 1: Continued.

\begin{tabular}{|c|c|c|c|c|c|c|c|c|}
\hline \multirow{3}{*}{ Problem } & \multirow{3}{*}{$n$} & \multirow{3}{*}{$x_{0}$} & \multicolumn{6}{|c|}{$\mathrm{NF} / \mathrm{NJ}$} \\
\hline & & & \multirow{2}{*}{ AELM } & \multicolumn{2}{|c|}{$\begin{array}{l}\text { Algorithm with } \\
\qquad \delta=1\end{array}$} & \multicolumn{3}{|c|}{ Algorithm with $\delta=2$} \\
\hline & & & & $\theta=0$ & $\theta=0.5$ & $\theta=0$ & $\theta=0.5$ & $\theta=1$ \\
\hline \multirow{18}{*}{ Discrete integral equation } & \multirow{6}{*}{30} & -100 & $16 / 16$ & $16 / 16$ & $16 / 16$ & $16 / 16$ & $16 / 16$ & $16 / 16$ \\
\hline & & -10 & $11 / 11$ & $11 / 11$ & $11 / 11$ & $11 / 11$ & $11 / 11$ & $11 / 11$ \\
\hline & & -1 & $9 / 9$ & $9 / 9$ & $9 / 9$ & $9 / 9$ & $9 / 9$ & $9 / 9$ \\
\hline & & 1 & $7 / 7$ & $7 / 7$ & $7 / 7$ & $6 / 6$ & $6 / 6$ & $6 / 6$ \\
\hline & & 10 & $11 / 11$ & $11 / 11$ & $11 / 11$ & $11 / 11$ & $11 / 11$ & $11 / 11$ \\
\hline & & 100 & $10 / 10$ & $10 / 10$ & $10 / 10$ & $10 / 10$ & $10 / 10$ & $10 / 10$ \\
\hline & \multirow{6}{*}{100} & -100 & $16 / 16$ & $16 / 16$ & $16 / 16$ & $16 / 16$ & $16 / 16$ & $16 / 16$ \\
\hline & & -10 & $12 / 12$ & $12 / 12$ & $12 / 12$ & $12 / 12$ & $12 / 12$ & $12 / 12$ \\
\hline & & -1 & $9 / 9$ & $10 / 10$ & $10 / 10$ & $9 / 9$ & $9 / 9$ & 9/9 \\
\hline & & 1 & $8 / 8$ & $7 / 7$ & $8 / 8$ & $7 / 7$ & $7 / 7$ & $7 / 7$ \\
\hline & & 10 & $12 / 12$ & $11 / 11$ & $11 / 11$ & $11 / 11$ & $11 / 11$ & $12 / 12$ \\
\hline & & 100 & $10 / 10$ & $10 / 10$ & $10 / 10$ & $10 / 10$ & $10 / 10$ & $10 / 10$ \\
\hline & \multirow{6}{*}{500} & -100 & $17 / 17$ & $17 / 17$ & $17 / 17$ & $17 / 17$ & $17 / 17$ & $17 / 17$ \\
\hline & & -10 & $13 / 13$ & $13 / 13$ & $13 / 13$ & $13 / 13$ & $13 / 13$ & $13 / 13$ \\
\hline & & -1 & $11 / 11$ & $11 / 11$ & $11 / 11$ & $11 / 11$ & $10 / 10$ & $10 / 10$ \\
\hline & & 1 & $9 / 9$ & $8 / 8$ & $9 / 9$ & $8 / 8$ & $8 / 8$ & $8 / 8$ \\
\hline & & 10 & $12 / 12$ & $12 / 12$ & $12 / 12$ & $12 / 12$ & $12 / 12$ & $12 / 12$ \\
\hline & & 100 & $10 / 10$ & $10 / 10$ & $10 / 10$ & $10 / 10$ & $10 / 10$ & $10 / 10$ \\
\hline
\end{tabular}

TABLE 2: Numerical results for singular nonlinear equations with $\operatorname{rank}\left(F^{\prime}\left(x^{*}\right)=n-1\right)$.

\begin{tabular}{|c|c|c|c|c|c|}
\hline \multirow{2}{*}{ Problem } & \multirow{2}{*}{$n$} & \multirow{2}{*}{$x_{0}$} & \multicolumn{3}{|c|}{ Iters/fnorm/trust/EI } \\
\hline & & & AELM (times: 3.63 ) & $\delta=1($ times: 7.47$)$ & $\delta=2($ times: 3.76$)$ \\
\hline \multirow{3}{*}{ Rosenbrock } & \multirow{3}{*}{2} & 1 & $16 / 1.5876 e-07 / 1 / 1.044$ & $16 / 2.3984 e-07 / 1 / 1.044$ & $16 / 2.3152 e-07 / 1 / 1.071$ \\
\hline & & 10 & $18 / 1.199 e-07 / 1 / 1.038$ & $18 / 1.211 e-07 / 1 / 1.038$ & $18 / 1.2102 e-07 / 1 / 1.0628$ \\
\hline & & 100 & $21 / 1.3601 e-07 / 1 / 1.034$ & $21 / 1.3604 e-07 / 1 / 1.034$ & $21 / 1.3603 e-07 / 1 / 1.053$ \\
\hline \multirow{3}{*}{ Powell badly } & \multirow{3}{*}{2} & 1 & $40 / 2.0905 e-07 / 1 / 1.017$ & $40 / 6.4701 e-07 / 1 / 1.017$ & $40 / 4.8829 e-07 / 1 / 1.027$ \\
\hline & & 10 & $25 / 3.4159 e-07 / 1 / 1.028$ & $25 / 3.6737 e-07 / 1 / 1.028$ & $25 / 3.0308 e-07 / 1 / 1.045$ \\
\hline & & 100 & $30 / 3.4445 e-07 / 1 / 1.023$ & $30 / 3.1265 e-07 / 1 / 1.023$ & $30 / 3.0929 e-07 / 1 / 1.037$ \\
\hline \multirow{3}{*}{ Wood } & \multirow{3}{*}{4} & 1 & $16 / 1.5876 e-07 / 1 / 1.044$ & $16 / 2.0905 e-07 / 1 / 1.044$ & $16 / 2.0897 e-07 / 1 / 1.071$ \\
\hline & & 10 & $19 / 1.926 e-07 / 1 / 1.037$ & $19 / 1.9262 e-07 / 1 / 1.037$ & $19 / 1.9262 e-07 / 1 / 1.059$ \\
\hline & & 100 & $22 / 2.8391 e-07 / 1 / 1.031$ & $22 / 2.8391 \mathrm{e} e-07 / 1 / 1.032$ & $22 / 2.8391 e-07 / 1 / 1.050$ \\
\hline \multirow{3}{*}{ Helical valley } & \multirow{3}{*}{3} & 1 & $9 / 9.8336 e-07 / 0 / 1.007$ & $9 / 1.5533 e-09 / 0 / 1.007$ & $96 / 1.4071 e-09 / 0 / 1.128$ \\
\hline & & 10 & $8 / 2.7959 e-07 / 0 / 1.090$ & $8 / 7.0428 e-07 / 0 / 1.090$ & $9 / 8.3791 e-14 / 0 / 1.128$ \\
\hline & & 100 & $8 / 2.0685 e-07 / 0 / 1.090$ & $8 / 1.2902 e-08 / 0 / 1.090$ & $8 / 1.1432 e-08 / 0 / 1.013$ \\
\hline \multirow{3}{*}{ Brown almost-linear } & \multirow{3}{*}{10} & 1 & $8 / 7.2842 e-07 / 1 / 1.090$ & $8 / 7.3653 e-05 / 1 / 1.090$ & $8 / 7.4202 e-05 / 1 / 1.013$ \\
\hline & & 10 & $23 / 7.9915 e-07 / 1 / 1.030$ & $23 / 7.9915 e-05 / 1 / 1.030$ & $23 / 7.9915 e-05 / 1 / 1.048$ \\
\hline & & 100 & $45 / 6.5326 e-07 / 1 / 1.015$ & $45 / 6.5326 e-05 / 1 / 1.015$ & $45 / 6.5326 e-05 / 11.024$ \\
\hline \multirow{3}{*}{ Discrete boundary value } & \multirow{3}{*}{10} & 1 & $3 / 0.00016727 / 0 / 1.259$ & $3 / 0.00016758 / 0 / 1.259$ & $4 / 1.1606 e-05 / 0 / 1.316$ \\
\hline & & 10 & $11 / 5.6012 e-07 / 0 / 1.064$ & $10 / 1.3863 e-05 / 0 / 1.071$ & $9 / 1.1763 e-05 / 0 / 1.129$ \\
\hline & & 100 & $9 / 9.2906 e-07 / 0 / 1.079$ & $9 / 8.9129 e-06 / 0 / 1.079$ & $9 / 8.7568 e-06 / 0 / / 1.129$ \\
\hline \multirow{3}{*}{ Discrete integral equation } & \multirow{3}{*}{30} & 1 & $7 / 9.5122 e-07 / 1 / 1.103$ & $7 / 8.4521 e-06 / 1 / 1.103$ & $6 / 1.1785 e-05 / 1 / 1.001$ \\
\hline & & 10 & $11 / 9.8059 e-07 / 1 / 1.064$ & $11 / 8.1669 e-06 / 1 / 1.064$ & $11 / 7.3177 e-06 / 1 / 1.002$ \\
\hline & & 100 & $10 / 1.0601 e-07 / 0 / 1.071$ & $10 / 1.0609 e-09 / 0 / 1.071$ & $10 / 1.0606 e-09 / 0 / 1.116$ \\
\hline \multirow{3}{*}{ Trigonometric } & \multirow{3}{*}{10} & 1 & $8 / 5.2977 e-07 / 0 / 1.008$ & $8 / 2.4187 e-07 / 0 / 1.008$ & $804 / 6.2122 e-09 / 1 / 1.001$ \\
\hline & & 10 & $19 / 2.3813 e-07 / 0 / 1.037$ & $-/ 19.064 / 0 / 1$ & $540 / 2.7155 e-09 / 1 / 1.002$ \\
\hline & & 100 & $-/ 46.227 e-07 / 0 / 1$ & $-/ 29.583 / 0 / 1$ & $19 / 62.146 / 1 / 1$ \\
\hline \multirow{3}{*}{ Variably dimensioned function } & \multirow{3}{*}{10} & 1 & $14 / 2.2087 e-07 / 0 / 1.050$ & $14 / 2.2087 e-05 / 1 / 1.050$ & $14 / 2.2087 e-05 / 1 / 1.081$ \\
\hline & & 10 & $16 / 1.1269 e-07 / 1 / 1.044$ & $16 / 1.1269 e-05 / 1 / 1.044$ & $16 / 1.1269 e-05 / 1 / 1.071$ \\
\hline & & 100 & $19 / 3.702 e-07 / 1 / 1.037$ & $19 / 3.702 e-05 / 1 / 1.037$ & $19 / 3.702 e-05 / 1 / 1.059$ \\
\hline \multirow{3}{*}{ Broyden tridiagonal } & \multirow{3}{*}{30} & 1 & $9 / 2.2916 e-07 / 1 / 1.007$ & $9 / 2.4133 e-05 / 1 / 1.007$ & $9 / 2.3888 e-05 / 1 / 1.129$ \\
\hline & & 10 & $14 / 1.419 e-05 / 1 / 1.050$ & $14 / 1.419 e-05 / 1 / 1.050$ & $14 / 1.419 e-05 / 1 / 1.081$ \\
\hline & & 100 & $17 / 2.5157 e-05 / 1 / 1.041$ & $17 / 2.5157 e-07 / 1 / 1.041$ & $17 / 2.5157 e-05 / 1 / 1.066$ \\
\hline \multirow{3}{*}{ Broyden banded } & \multirow{3}{*}{30} & 1 & $12 / 4.2271 e-07 / 1 / 1.059$ & $12 / 4.2313 e-06 / 1 / 1.059$ & $12 / 4.2305 e-06 / 1 / 1.095$ \\
\hline & & 10 & $18 / 4.5992 e-07 / 1 / 1.039$ & $18 / 4.5992 e-06 / 1 / 1.039$ & $18 / 4.5992 e-06 / 1 / 1.062$ \\
\hline & & 100 & $24 / 2.7182 e-07 / 1 / 1.029$ & $24 / 2.7182 e-06 / 1 / 1.029$ & $24 / 2.7182 e-06 / 1 / 1.046$ \\
\hline
\end{tabular}


TABLE 3: Results on singular nonlinear equations with rank $\left(F^{\prime}\left(x^{*}\right)=n-2\right.$.

\begin{tabular}{|c|c|c|c|c|c|c|c|c|}
\hline \multirow{3}{*}{ Problem } & \multirow{3}{*}{$n$} & \multirow{3}{*}{$x_{0}$} & \multicolumn{6}{|c|}{$\mathrm{NF} / \mathrm{NJ}$} \\
\hline & & & \multirow{2}{*}{ AELM } & \multicolumn{2}{|c|}{ Algorithm with $\delta=1$} & \multicolumn{3}{|c|}{ Algorithm with $\delta=2$} \\
\hline & & & & $\theta=0$ & $\theta=0.5$ & $\theta=0$ & $\theta=0.5$ & $\theta=1$ \\
\hline \multirow{6}{*}{ Powell badly scaled } & \multirow{6}{*}{2} & -100 & $852 / 717$ & $275 / 250$ & $169 / 158$ & $1802 / 1521$ & $2585 / 2168$ & $366 / 318$ \\
\hline & & -10 & $76 / 64$ & $163 / 144$ & $69 / 61$ & $1595 / 1334$ & $3865 / 3226$ & $585 / 484$ \\
\hline & & -1 & $183 / 167$ & $97 / 79$ & $118 / 103$ & $2754 / 2296$ & $2585 / 2168$ & $341 / 279$ \\
\hline & & 1 & $1288 / 1076$ & $128 / 111$ & $127 / 105$ & - & $996 / 833$ & $711 / 591$ \\
\hline & & 10 & $3 / 3$ & $92 / 76$ & $3 / 3$ & $7 / 4$ & $7 / 4$ & $1043 / 873$ \\
\hline & & 100 & $3 / 3$ & $3 / 3$ & $3 / 3$ & $3 / 3$ & $3 / 3$ & $1954 / 1622$ \\
\hline \multirow{12}{*}{ Discrete boundary value } & \multirow{7}{*}{10} & -100 & $14 / 14$ & $14 / 14$ & $14 / 14$ & $14 / 14$ & $14 / 14$ & $14 / 14$ \\
\hline & & -10 & $11 / 11$ & $10 / 10$ & $11 / 11$ & $10 / 10$ & $10 / 10$ & $10 / 10$ \\
\hline & & -1 & $8 / 8$ & $8 / 8$ & $8 / 8$ & $7 / 7$ & $7 / 7$ & $7 / 7$ \\
\hline & & 1 & $3 / 3$ & $3 / 3$ & $3 / 3$ & $4 / 4$ & $4 / 4$ & $4 / 4$ \\
\hline & & 10 & $11 / 11$ & $10 / 10$ & $10 / 10$ & $9 / 9$ & $9 / 9$ & $9 / 9$ \\
\hline & & 100 & $10 / 10$ & $10 / 10$ & $10 / 10$ & $10 / 10$ & $10 / 10$ & $10 / 10$ \\
\hline & & -100 & $14 / 14$ & $13 / 13$ & $14 / 14$ & $13 / 13$ & $14 / 14$ & $14 / 14$ \\
\hline & \multirow{5}{*}{100} & -10 & $11 / 11$ & $9 / 9$ & $11 / 11$ & $8 / 8$ & $9 / 9$ & $9 / 9$ \\
\hline & & -1 & $6 / 6$ & $5 / 5$ & $5 / 5$ & $4 / 4$ & $4 / 4$ & $4 / 4$ \\
\hline & & 1 & $4 / 4$ & $4 / 4$ & $4 / 4$ & $3 / 3$ & $3 / 3$ & $3 / 3$ \\
\hline & & 10 & $7 / 7$ & $9 / 9$ & $7 / 7$ & $7 / 7$ & $8 / 8$ & $8 / 8$ \\
\hline & & 100 & $13 / 13$ & $12 / 12$ & $13 / 13$ & $11 / 11$ & $12 / 12$ & $13 / 13$ \\
\hline \multirow{12}{*}{ Discrete integral equation } & \multirow{6}{*}{30} & -100 & $16 / 16$ & $16 / 16$ & $16 / 16$ & $16 / 16$ & $16 / 16$ & $16 / 16$ \\
\hline & & -10 & $11 / 11$ & $11 / 11$ & $11 / 11$ & $11 / 11$ & $11 / 11$ & $11 / 11$ \\
\hline & & -1 & $9 / 9$ & $9 / 9$ & $9 / 9$ & $9 / 9$ & $9 / 9$ & $9 / 9$ \\
\hline & & 1 & $7 / 7$ & $7 / 7$ & $7 / 7$ & $6 / 6$ & $6 / 6$ & $6 / 6$ \\
\hline & & 10 & $11 / 11$ & $11 / 11$ & $11 / 11$ & $11 / 11$ & $11 / 11$ & $11 / 11$ \\
\hline & & 100 & $12 / 12$ & $12 / 12$ & $12 / 12$ & $12 / 12$ & $12 / 12$ & $12 / 12$ \\
\hline & \multirow{6}{*}{100} & -100 & $16 / 16$ & $16 / 16$ & $16 / 16$ & $16 / 16$ & $16 / 16$ & $16 / 16$ \\
\hline & & -10 & $12 / 12$ & $12 / 12$ & $12 / 12$ & $12 / 12$ & $12 / 12$ & $12 / 12$ \\
\hline & & -1 & $10 / 10$ & $10 / 10$ & $10 / 10$ & $9 / 9$ & $9 / 9$ & $9 / 9$ \\
\hline & & 1 & $8 / 8$ & $7 / 7$ & $8 / 8$ & $7 / 7$ & $7 / 7$ & $7 / 7$ \\
\hline & & 10 & $12 / 12$ & $11 / 11$ & $11 / 11$ & $11 / 11$ & $11 / 11$ & $12 / 12$ \\
\hline & & 100 & $14 / 14$ & $14 / 14$ & $14 / 14$ & $14 / 14$ & $14 / 14$ & $14 / 14$ \\
\hline
\end{tabular}

TABLE 4: Numerical results for singular nonlinear equations with rank $\left(F^{\prime}\left(x^{*}\right)=n-2\right)$.

\begin{tabular}{|c|c|c|c|c|c|}
\hline \multirow{2}{*}{ Problem } & \multirow{2}{*}{$n$} & \multirow{2}{*}{$x_{0}$} & \multicolumn{3}{|c|}{ Iters/fnorm/trust/EI } \\
\hline & & & AELM (times: 4.655) & $\delta=1($ times: 3.374$)$ & $\delta=2($ times: 3.614$)$ \\
\hline \multirow{3}{*}{ Rosenbrock } & \multirow{3}{*}{2} & 1 & $11 / 4.6197 e-05 / 0 / 1.065$ & $11 / 4.6202 e-05 / 0 / 1.065$ & $11 / 4.6202 e-05 / 0 / 1.105$ \\
\hline & & 10 & $13 / 0.00010074 / 0 / 1.054$ & $13 / 0.00010074 / 0 / 1.054$ & $13 / 0.00010074 / 0 / 1.088$ \\
\hline & & 100 & $17 / 3.4089 e-05 / 0 / 1.041$ & $17 / 3.4089 e-05 / 0 / 1.041$ & $21 / 63 / 3.4089 e-05 / 0 / 1.066$ \\
\hline \multirow{3}{*}{ Powell badly } & \multirow{3}{*}{2} & 1 & $1288 / 1.0433 e-07 / 0 / 1.000$ & $127 / 4.9595 e-07 / 1 / 1.008$ & $996 / 41.8112 e-07 / 0 / 1.001$ \\
\hline & & 10 & $3 / 3.3583 e-05 / 0 / 1.259$ & $3 / 3.3562 e-05 / 0 / 1.028$ & $7 / 1.6053 e-05 / 0 / 1.169$ \\
\hline & & 100 & $3 / 0.0099779 / 0 / 1.259$ & 3/0.009978/0/1.023 & 3/0.009978/0/1.442 \\
\hline \multirow{3}{*}{ Wood } & \multirow{3}{*}{4} & 1 & $14 / 3.2876 e-06 / 0 / 1.050$ & $14 / 3.2877 e-06 / 0 / 1.081$ & $14 / 3.2877 e-06 / 0 / 1.081$ \\
\hline & & 10 & $17 / 3.0842 e-06 / 0 / 1.041$ & $17 / 3.0842 e-06 / 0 / 1.041$ & $17 / 3.0842 e-06 / 1 / 1.066$ \\
\hline & & 100 & $20 / 4.5433 e-06 / 0 / 1.035$ & $20 / 4.5433 e-06 / 0 / 1.035$ & $20 / 4.5433 e-06 / 0 / 1.056$ \\
\hline \multirow{3}{*}{ Helical valley } & \multirow{3}{*}{3} & 1 & $13 / 5.9421 e-06 / 1 / 1.054$ & $13 / 5.9553 e-06 / 0 / 1.054$ & $13 / 5.9694 e-06 / 0 / 1.088$ \\
\hline & & 10 & $14 / 2.087 e-06 / 1 / 1.050$ & $14 / 2.0897 e-06 / 0 / 1.050$ & $14 / 2.0934 e-06 / 1 / 1.081$ \\
\hline & & 100 & $18 / 3.0231 e-06 / 1 / 1.039$ & $18 / 3.1777 e-06 / 0 / 1.039$ & $18 / 3.3522 e-06 / 1 / 1.062$ \\
\hline \multirow{3}{*}{ Brown almost-linear } & \multirow{3}{*}{10} & 1 & $8 / 7.2842 e-05 / 1 / 1.147$ & $8 / 7.3653 e-05 / 1 / 1.090$ & $8 / 7.4202 e-05 / 0 / 1.147$ \\
\hline & & 10 & $23 / 7.9915 e-05 / 1 / 1.030$ & $23 / 7.9915 e-05 / 1 / 1.030$ & $23 / 7.9915 e-05 / 1 / 1.048$ \\
\hline & & 100 & $45 / 6.5326 e-05 / 1 / 1.015$ & $45 / 6.5326 e-05 / 1 / 1.015$ & $45 / 6.5325 e-05 / 1 / 1.024$ \\
\hline \multirow{3}{*}{ Discrete boundary value } & \multirow{3}{*}{10} & 1 & $3 / 0.0001676 / 0 / 1.259$ & $3 / 0.00016795 / 0 / 1.259$ & $4 / 1.1572 e-05 / 0 / 1.316$ \\
\hline & & 10 & $11 / 5.5963 e-06 / 0 / 1.065$ & $10 / 48 / 1.383 e-05 / 0 / 1.065$ & $9 / 1.2359 e-05 / 0 / 1.129$ \\
\hline & & 100 & $10 / 6.7629 e-08 / 0 / 1.071$ & $10 / 6.3555 e-08 / 0 / 1.071$ & $10 / 6.1475 e-08 / 0 / / 1.116$ \\
\hline \multirow{3}{*}{ Discrete integral equation } & \multirow{3}{*}{30} & 1 & $7 / 9.5122 e-06 / 1 / 1.104$ & $7 / 8.4521 e-06 / 1 / 1.104$ & $6 / 1.1785 e-05 / 1 / 1.200$ \\
\hline & & 10 & $11 / 9.806 e-06 / 1 / 1.065$ & $11 / 8.167 e-06 / 1 / 1.065$ & $11 / 7.3178 e-06 / 1 / 1.105$ \\
\hline & & 100 & $12 / 1.3149 e-07 / 0 / 1.059$ & $12 / 11.8382 e-07 / 0 / 1.059$ & $12 / 1.137 e-09 / 0 / 1.095$ \\
\hline
\end{tabular}


TABle 4: Continued.

\begin{tabular}{|c|c|c|c|c|c|}
\hline Problem & $n$ & $x_{0}$ & AELM (times: 4.655 ) & $\begin{array}{l}\text { Iters/fnorm/trust/EI } \\
\delta=1 \text { (times: } 3.374 \text { ) }\end{array}$ & $\delta=2$ (times: 3.614$)$ \\
\hline Trigonometric & 10 & $\begin{array}{c}1 \\
10 \\
100 \\
\end{array}$ & $\begin{array}{c}13 / 2.2087 e-05 / 1 / 1.054 \\
31 / 1.113 e-09 / 0 / 1.022 \\
-/ 15.261 / 0 / 1 \\
\end{array}$ & $\begin{array}{c}13 / 9.9955 e-06 / 0 / 1.054 \\
22 / 2.4389 e-08 / 0 / 1.032 \\
1 / 120.79 / 0 / 1 \\
\end{array}$ & $\begin{array}{c}14 / 7.3496 e-06 / 1 / 1.001 \\
16 / 1.8831 e-07 / 0 / 1.002 \\
19 / 48 / 170.57 / 0 / 1\end{array}$ \\
\hline Variably dimensioned function & 10 & $\begin{array}{c}1 \\
10 \\
100 \\
\end{array}$ & $\begin{array}{c}14 / 2.2087 e-05 / 1 / 1.050 \\
16 / 1.1269 e-05 / 0 / 1.044 \\
19 / 3.702 e-05 / 0 / 1.037 \\
\end{array}$ & $\begin{array}{c}14 / 2.2087 e-05 / 1 / 1.050 \\
16 / 1.1269 e-05 / 0 / 1.044 \\
19 / 3.702 e-05 / 0 / 1.037\end{array}$ & $\begin{array}{c}14 / 2.2087 e-05 / 1 / 1.081 \\
16 / 1.1269 e-05 / 0 / 1.071 \\
19 / 3.702 e-05 / 0 / 1.059\end{array}$ \\
\hline Broyden tridiagonal & 30 & $\begin{array}{c}1 \\
10 \\
100 \\
\end{array}$ & $\begin{array}{c}9 / 2.294 e-05 / 1 / 1.080 \\
14 / 1.4189 e-05 / 1 / 1.050 \\
17 / 2.5157 e-05 / 1 / 1.041 \\
\end{array}$ & $\begin{array}{c}9 / 2.4133 e-05 / 1 / 1.007 \\
14 / 1.419 e-05 / 1 / 1.050 \\
17 / 2.5157 e-07 / 1 / 1.041 \\
\end{array}$ & $\begin{array}{c}9 / 2.3903 e-05 / 1 / 1.129 \\
14 / 1.419 e-05 / 1 / 1.081 \\
17 / 2.5157 e-05 / 1 / 1.066 \\
\end{array}$ \\
\hline Broyden banded & 30 & $\begin{array}{c}1 \\
10 \\
100 \\
\end{array}$ & $\begin{array}{l}12 / 4.2271 e-07 / 1 / 1.059 \\
18 / 4.5992 e-07 / 1 / 1.039 \\
24 / 2.7182 e-07 / 1 / 1.029\end{array}$ & $\begin{array}{c}12 / 4.2313 e-06 / 1 / 1.059 \\
18 / 4.2297 e-06 / 11.039 \\
24 / 2.7182 e-06 / 1 / 1.029\end{array}$ & $\begin{array}{l}12 / 4.2305 e-06 / 1 / 1.095 \\
18 / 4.5984 e-06 / 1 / 1.062 \\
24 / 2.7178 e-06 / 1 / 1.046\end{array}$ \\
\hline
\end{tabular}

where $A \in \mathbf{R}^{n \times k}(1 \leq k \leq n)$ has full column rank, and $F\left(x^{*}\right)=0$. It is certain that

$$
\bar{J}\left(x^{*}\right)=J\left(x^{*}\right)\left(I-A\left(A^{T} A\right)^{-1} A^{T}\right),
$$

where test problems $F(x)$ are nonsingular test functions from [20]. We take $A=[1,1, \ldots, 1]^{T} \in \mathbf{R}^{n \times 1}, \operatorname{rank}$ of $\bar{J}\left(x^{*}\right)$ as $n-1$, and choose

$$
A=\left[\begin{array}{cccc}
1 & 1 & \cdots & 1 \\
1 & -1 & \cdots & \pm 1
\end{array}\right],
$$

rank of $\bar{J}\left(x^{*}\right)$ as $n-2$.

We set the following parameters in the algorithms: $p_{0}=10^{-4}, p_{1}=0.25, p_{1}=0.75, N_{0}=5, \mu_{1}=1, m=10^{-8}$, $\theta=0,0.5$ or 1 , and $\delta=1$ or 2 . The algorithms are terminated when $\left\|J_{k}^{T} F_{k}\right\| \leq 10^{-5}$ or the number of iterates exceeds $10^{4}$. When $\theta=1$ and $\delta=1$, Algorithm 1 is reduced to the AELM.

By numerical experiments, we find the numerical results of Algorithm 1 are the same as the numerical results of AELM in some functions. So we only list the results of other experiments in the following tables. Further, we adopt the efficiency index defined as EI in [21] to compare the performance of algorithm AELM and Algorithm 1. The results of the four experiments with $\operatorname{rank} J\left(x^{*}\right)=n-1$ are shown in Tables 1 and 2, and the results of the four experiments with $\operatorname{rank} J\left(x^{*}\right)=n-2$ are shown in Tables 3 and 4 , respectively. We use six starting points $\pm 100 x_{0}, \pm 10 x_{0}$, and $\pm x_{0}$ for each test problem, where $x_{0}$ is suggested in [20].

(i) NF stands for the quantity of function calculations

(ii) NJ stands for the quantity of Jacobian calculations

(iii) '-' indicates that the iteration number is more than $10^{4}$

(iv) E.I. $=\rho^{1 / N F}$, where $\rho$ is the convergence order of algorithm.

It is shown in Table 1 that when $\theta=0.5$ and $\delta=2$, the effect of Algorithm 1 is obviously better than that of AELM. Algorithm 1 wins $40.5 \%$ of the numerical results while AELM wins $2.38 \%$, and $57.1 \%$ of the two algorithms have the same results. The advantage of Algorithm 1 is not obvious when $\theta=0.5$ and $\delta=1$. Algorithm 1 can win $19 \%$ of the numerical results while AELM win 7.14\%, and 73.8\% of two algorithms have the same result.

Table 3 shows that when $\theta=0.5$ and $\delta=1$, Algorithm 1 and AELM have the best experimental results. Algorithm 1 win $23.3 \%$ of the numerical results, and $76.6 \%$ of the two algorithms has the same result. The advantage of Algorithm 1 is not obvious when $\theta=0.5$ and $\delta=2$. Algorithm 1 wins $40 \%$ of the numerical results while AELM wins $20 \%$, and $40 \%$ of the two algorithms has the same results.

Further, we adopt the EI and let $\theta=0.5$ in the experiment. Tables 2 and 4 show that when $\delta=1$, the experimental data EI of AELM and Algorithm 1 are similar, but when $\delta=1$, the EI of Algorithm 1 is obviously larger than that of AELM. In addition, in terms of the experimental time, except when ranking $\left(F^{\prime}\left(x^{*}\right)=n-2\right)$ and $\delta=1$, the execution time of Algorithm 1 is longer than that of AELM. In other cases, the execution time of Algorithm 1 is close to or less than that of AELM.

In general, it is shown that for most test problems, Algorithm 1 performs better than AELM. So it can indicate that Algorithm 1 is more efficient than AELM to solve systems of nonlinear equations.

\section{Conclusion}

In this paper, we propose a new LM algorithm by modifying the LM parameter for systems of nonlinear equations. Through numerical experiments, we find the calculation amounts of Algorithm 1 smaller than AELM in the case where $\theta$ and $\delta$ take some suitable value, which shows the effectiveness of the new Algorithm 1. Under some conditions, the global convergence of the new LM method is proved, and the local convergence of the new LM method is shown to be at least superlinear. Numerical results show that the new algorithm is efficient.

\section{Data Availability}

No data were used to support the findings of this study.

\section{Conflicts of Interest}

The authors declare that they have no conflicts of interest. 


\section{Acknowledgments}

This research was supported by the Natural Science Foundation of the Anhui Higher Education Institutions under Grant nos. KJ2020ZD008 and KJ2019A0604, by the Natural Science Foundation of Anhui Province under no. 2108085MF204, and by the Abroad Visiting of Excellent Young Talents in Universities of Anhui Province under Grant no. GXGWFX2019022.

\section{References}

[1] J. Nocedal and S. J. Wright, Numerical Optimization, Springer, New York, NY, USA, 1999.

[2] K. Levenberg, "A method for the solution of certain nonlinear problems in least squares," Quarterly of Applied Mathematics, vol. 2, no. 2, pp. 164-168, 1944.

[3] D. W. Marquardt, "An algorithm for least-squares estimation of nonlinear parameters," Journal of the Society for Industrial and Applied Mathematics, vol. 11, no. 2, pp. 431-441, 1963.

[4] N. Yamashita and M. Fukushima, "On the rate of convergence of the levenberg-marquardt method," in Topics in Numerical Analysis, pp. 239-249, Springer, Vienna, AS, USA, 2001.

[5] J.-Y. Fan and Y.-X. Yuan, "On the quadratic convergence of the levenberg-marquardt method without nonsingularity assumption," Computing, vol. 74, no. 1, pp. 23-39, 2005.

[6] K. Amini, F. Rostami, and G. Caristi, "An efficient LevenbergMarquardt method with a new LM parameter for systems of nonlinear equations," Optimization, vol. 67, no. 5, pp. 637-650, 2018.

[7] A. O. Umar, I. M. Sulaiman, M. Mamat, M. Y. Waziri, and N. Zamri, "On damping parameters of Levenberg-Marquardt algorithm for nonlinear least square problems," Journal of Physics: Conference Series, vol. 1734, Article ID 012018, 2021.

[8] H. Wang and J. Fan, "Convergence rate of the LevenbergMarquardt method under Hölderian local error bound," Optimization Methods and Software, vol. 35, no. 4, pp. 767786, 2020.

[9] C. Ma and L. Jiang, "Some research on Levenberg-Marquardt method for the nonlinear equations," Applied Mathematics and Computation, vol. 184, no. 2, pp. 1032-1040, 2007.

[10] J. Fan, J. Huang, and J. Pan, "An adaptive multi-step levenberg-marquardt method," Journal of Scientific Computing, vol. 78, no. 1, pp. 531-548, 2019.

[11] K. Amini and F. Rostami, "A modified two steps LevenbergMarquardt method for nonlinear equations," Journal of Computational and Applied Mathematics, vol. 288, pp. 341$350,2015$.

[12] L. Chen and Y. Ma, "Shamanskii-like Levenberg-Marquardt method with a new line search for systems of nonlinear equations," Journal of Systems Science and Complexity, vol. 33, no. 5, pp. 1694-1707, 2020.

[13] L. Grippo, F. Lampariello, and S. Lucidi, "A nonmonotone line search technique for Newton's method," SIAM Journal on Numerical Analysis, vol. 23, no. 4, pp. 707-716, 1986.

[14] M. Ahookhosh and K. Amini, "An efficient nonmonotone trust-region method for unconstrained optimization," $\mathrm{Nu}$ merical Algorithms, vol. 59, no. 4, pp. 523-540, 2012.

[15] M. Ahookhosh and K. Amini, "A Nonmonotone trust region method with adaptive radius for unconstrained optimization problems," Computers and Mathematics with Applications, vol. 60, no. 3, pp. 411-422, 2010.
[16] M. J. D. Powell, "An iterative method for finding stationary values of a function of several variables," The Computer Journal, vol. 5, no. 2, pp. 147-151, 1962.

[17] W. Zhou, "On the convergence of the modified LevenbergMarquardt method with a nonmonotone second order Armijo type line search," Journal of Computational and Applied Mathematics, vol. 239, pp. 152-161, 2013.

[18] G. W. Stewart and J.-G. Sun, Matrix Perturbation Theory, Academic Press, Cambridge, MA, USA, 1990.

[19] R. B. Schnabel and P. D. Frank, "Tensor methods for nonlinear equations," SIAM Journal on Numerical Analysis, vol. 21, no. 5, pp. 815-843, 1984.

[20] J. J. Moré, B. S. Garbow, and K. E. Hillstrom, "Testing unconstrained optimization software," ACM Transactions on Mathematical Software, vol. 7, no. 1, pp. 17-41, 1981.

[21] S. Qasim, Z. Ali, F. Ahmad et al., "Solving systems of nonlinear equations when the nonlinearity is expensive," Computers and Mathematics with Applications, vol. 71, no. 7, pp. 1464-1478, 2016. 